Review

\title{
Lung cancer in young adults aged 35 years or younger: $A$ full-scale analysis and review
}

\author{
Bailong Liu*, Xiaoyue Quan*, Changgen $\mathrm{Xu}^{*}$, Jincai Lv, Cheng Li, Lihua Dong ${ }^{\circledR}$, Min Liu ${ }^{\varpi}$ \\ Department of Radiation Oncology, The First Hospital of Jilin University, 71 Xinmin Street, Changchun, 130021, China \\ "These authors contributed equally to this article. \\ $\triangle$ Corresponding authors: Lihua Dong, MD, PhD. Department of Radiation Oncology, The First Hospital of Jilin University, 71 Xinmin Street, Changchun, \\ 130021, China. Phone: 86 158-4307-3216 E-mail: drlhdong@163.com. Min Liu, MD, PhD. Department of Radiation Oncology, The First Hospital of Jilin \\ University, 71 Xinmin Street, Changchun, 130021, China. Phone: 86 158-0430-2751 E-mail: Imin99@jlu.edu.cn \\ (c) Ivyspring International Publisher. This is an open access article distributed under the terms of the Creative Commons Attribution (CC BY-NC) license \\ (https://creativecommons.org/licenses/by-nc/4.0/). See http://ivyspring.com/terms for full terms and conditions.
}

Received: 2018.05.26; Accepted: 2019.05.01; Published: 2019.06.09

\begin{abstract}
Objectives: Lung cancer in young adults is a distinct disease with particular socioeconomic implications. This study aimed to clarify the clinicopathological characteristics, best interventions, and outcomes of this distinctive entity.

Methods: A retrospective review of patients with lung cancer was performed in our institute from January 2010 to June 2017. Young adults were defined as between 18 and 35 years old. Demographic, clinicopathological, therapeutic, and prognostic data were systematically analyzed.

Results: From a total of 8734 patients, 120 (1.37\%) were young adults, of which 82 with complete hospital records were included in this study. A high proportion had adenocarcinoma (45\%) and late-stage disease ( $49.21 \%$ stage IV at diagnosis). Pleura (38.71\%) were the most common metastatic site, followed by bone (35.48\%) and lung $(25.81 \%)$. The majority $(68 \%)$ had single organ metastasis. Young patients had an increased frequency of gene mutations. Among the 18 patients for whom epidermal growth factor receptor (EGFR) and anaplastic lymphoma kinase (ALK) status was determined, 10 had sensitive EGFR mutations while 5 had ALK rearrangement; only 3 patients were driver gene mutation-negative. The 1-year overall survival (OS) rate was $62.31 \%$ and the 3 - and 5 -year survival rates were both $53.31 \%$; median OS was not achieved (range, 3-86 months). Male sex, negative or unknown gene mutation status, stage IV, and squamous or small cell lung cancer were associated with poor prognosis (OS) in early-onset lung cancer.

Conclusions: Lung cancer in young adults is distinctive, with adenocarcinoma and stage IV at presentation being predominant characteristics. Gene mutation assessment should be mandatory in this subgroup due to the increased likelihood of positive driver gene alterations, as individualized targeted therapy may achieve superior outcomes.
\end{abstract}

Key words: Lung cancer, Young patients, Gene mutation, Combined treatment

\section{Introduction}

Lung cancer is the leading cause of mortality worldwide. In China, both incidence and mortality from lung cancer continue to increase sharply [1], posing a serious threat to public health. In 2015, lung cancer had the highest mortality rate (standardized mortality rate, 39.81/100,000) of any cancer in China [2]. Notably, research from Europe and Japan has shown a trend of increasing incidence of lung cancer in young adults $[3,4]$ and there is a pressing need to characterize the disease in this subgroup because of its distinct biological behavior, previous limited study, and particular socioeconomic implications. Previously, the cut-off age for early-onset lung cancer has ranged from 40 to 50 years [5]. Our study is the first to date to explore this distinctive disease in patients aged 18-35 years. Our overview will help to 
expand understanding of this unique subgroup of lung cancer and provide meaningful information to guide its optimal treatment and improve prognosis.

\section{Materials and Methods}

\section{Study population}

From January 2010 to June 2017, 8734 patients with lung cancer confirmed by pathological examination of either resection or biopsy samples in the First Hospital, Jilin University were reviewed. Among them, 120 adults aged 18-35 years were eligible for this study; however, 38 patients diagnosed in the outpatient service were excluded because of a lack of treatment records. Finally, 82 patients were included in this retrospective study. The following data were retrieved: age; sex; family history; personal history (including smoking and alcohol consumption); pathological tumor type; tumor location; tumor stage; gene mutation assessment; interventions, including surgery, chemotherapy, radiotherapy, and targeted management; and overall survival (OS). OS was defined as the interval from diagnosis to death or latest follow-up. The end date for last follow-up was April 30, 2018.

\section{Ethical considerations}

This study was approved by the Ethics Committee of the First Hospital of Jilin University.

\section{Statistical analysis}

Cumulative survival curves were generated by the Kaplan-Meier method and differences in survival rates compared using the log-rank (Mantel-Cox) and/or Gehan-Breslow-Wilcoxon tests. P-values < 0.05 were considered statistically significant.

\section{Results}

\section{Patient characteristics}

Lung cancer is exceedingly rare in young individuals, with an incidence of $1.37 \%(120 / 8734)$ in our registry, which was similar in both sexes (M:F ratio, 1.16:1), unlike some previous reports of higher rates in females than males [1]. The mean age at diagnosis was 31.39 years. The "31-35 years" age group comprised $69.51 \%$ of all enrolled patients, with the "20-30 years" age group making up $28.05 \%$. Only two patients were 18 years old at diagnosis. Family history was unremarkable in $98.68 \%$ of patients. Fifty-eight patients $(71.60 \%)$ stated that they did not smoke while 23 had a history of smoking, among which 6 were heavy smokers, with $\geq 15$ years of smoking history. Most patients (91.36\%) had no history of alcohol consumption. Regarding primary site, there was no predilection for either the left or right lung (L:R ratio, 0.82:1). Notably, nearly half of patients $(49.21 \%)$ with records of tumor stage were recorded as having metastatic disease at diagnosis (Table 1).

Table 1. Clinical features of lung cancer in young adults

\begin{tabular}{|c|c|c|}
\hline & $\mathrm{n}$ & $\%$ \\
\hline \multicolumn{3}{|c|}{$\operatorname{Sex}(n=82)$} \\
\hline Male & 44 & 53.66 \\
\hline Female & 38 & 46.34 \\
\hline \multicolumn{3}{|c|}{ Age $(n=82)$} \\
\hline \multicolumn{3}{|c|}{ Mean at diagnosis (years) 31.39} \\
\hline \multicolumn{3}{|c|}{ Range (years) $18-35$} \\
\hline $31-35$ & 57 & 69.51 \\
\hline $20-30$ & 23 & 28.05 \\
\hline$<20$ & 2 & 2.44 \\
\hline \multicolumn{3}{|c|}{ Family history $(\mathrm{n}=76)$} \\
\hline Yes & 1 & 1.32 \\
\hline No & 75 & 98.68 \\
\hline \multicolumn{3}{|c|}{ Smoking history $(\mathrm{n}=81)$} \\
\hline Yes & 23 & 28.40 \\
\hline No & 58 & 71.60 \\
\hline \multicolumn{3}{|c|}{ Alcohol consumption history $(\mathrm{n}=81)$} \\
\hline Yes & 7 & 8.64 \\
\hline No & 74 & 91.36 \\
\hline \multicolumn{3}{|c|}{ Primary sites $(n=82)$} \\
\hline Left & 37 & 45.12 \\
\hline Right & 45 & 54.88 \\
\hline \multicolumn{3}{|c|}{ Stage $(n=63)$} \\
\hline 0 & 1 & 1.59 \\
\hline I & 12 & 19.05 \\
\hline II & 6 & 9.52 \\
\hline III & 13 & 20.63 \\
\hline IV & 31 & 49.21 \\
\hline
\end{tabular}

Among metastatic sites, pleura were the most common. Of early-onset stage IV lung cancer patients, $38.71 \%$ suffered from malignant pleural effusion at presentation. Bone was the second most common metastatic site $(35.48 \%)$, followed by lung and liver $(25.81 \%$ and $12.90 \%$, respectively) (Table 2). Furthermore, $68 \%$ of patients with stage IV early-onset lung cancer at diagnosis had single organ involvement (i.e., oligometastasis), indicating that aggressive local and systemic management could contribute to better outcomes for such patients (Figure $1)$.

Table 2. Metastatic sites of young patients with lung cancer at diagnosis $(n=31)$

\begin{tabular}{lll}
\hline Metastasis sites & $\mathrm{n}$ & $\%$ \\
\hline Lung & 8 & 25.81 \\
Liver & 4 & 12.90 \\
Bone & 11 & 35.48 \\
Brain & 3 & 9.68 \\
Pleura & 12 & 38.71 \\
Pericardium & 1 & 3.23 \\
Spleen & 1 & 3.23 \\
Chest wall & 1 & 3.23 \\
Intraperitoneal sites & 1 & 3.23 \\
\hline
\end{tabular}




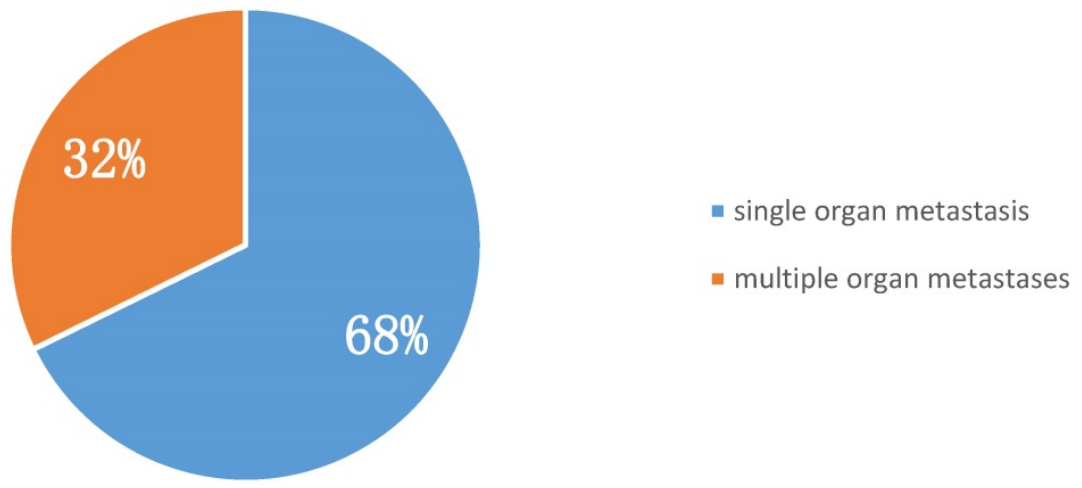

Figure 1. Proportion of single and multiple organ metastases in stage IV early-onset lung cancer at diagnosis $(n=31)$.

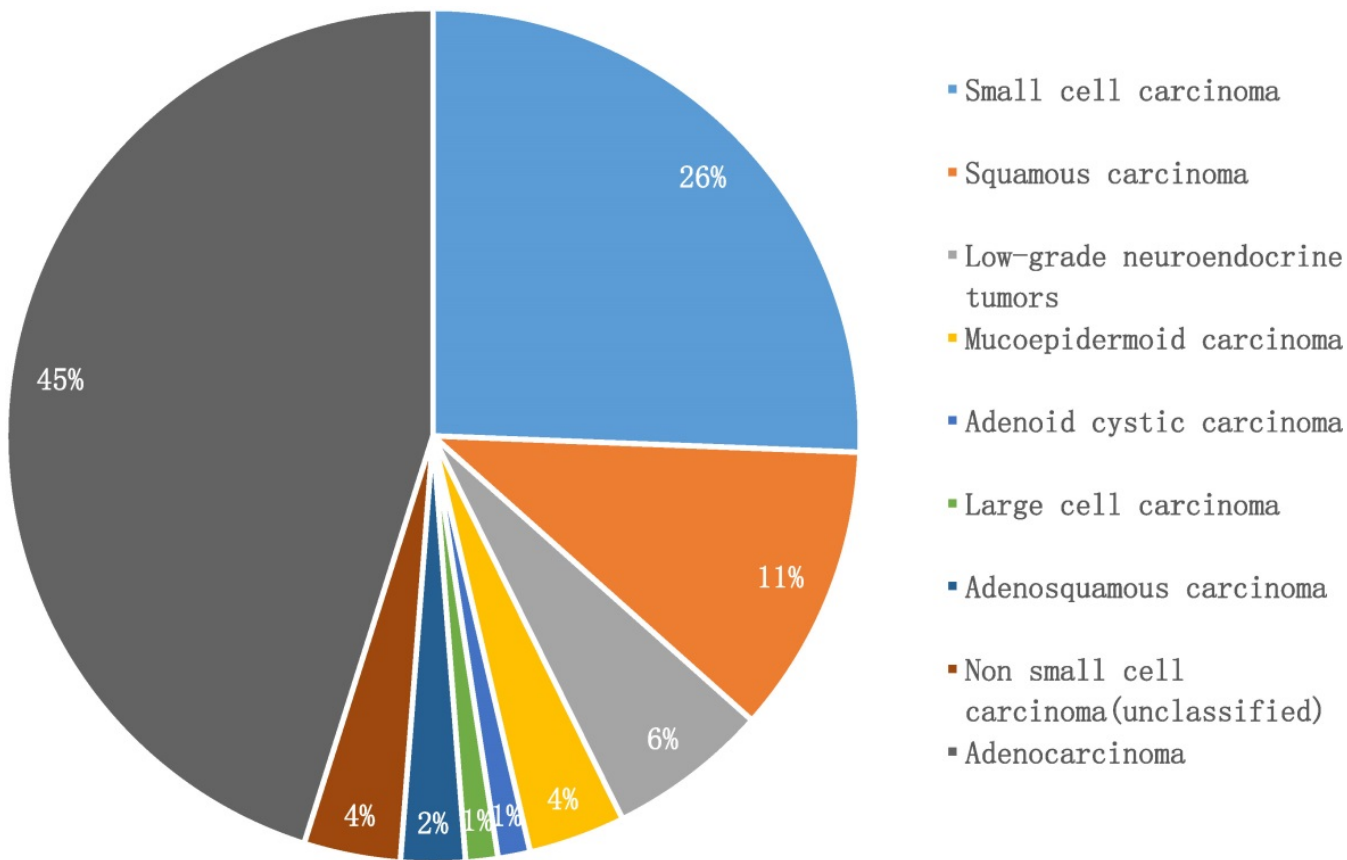

Figure 2. Histologic types in young adults with lung cancer $(n=82)$

\section{Pathological and molecular tests}

Adenocarcinoma was the predominant subtype in our cohort $(45 \%, \mathrm{n}=37)$, while small cell lung cancer accounted for $26 \%(n=21)$, followed by squamous carcinoma $(11 \%, n=9)$. Notably, low-grade neuroendocrine lung tumors comprised only $6 \%(n=$ 5). Infrequent pathologic subtypes included mucoepidermoid carcinoma $(\mathrm{n}=3)$, adenosquamous carcinoma $(n=2)$, adenoid cystic carcinoma $(n=1)$, and large cell carcinoma $(\mathrm{n}=1)$. Three patients were only classified as having non-small cell lung cancer, due to lack of further immunohistochemical examination (Figure 2).

A total of 53 patients had clear genetic status records, while genetic testing was not performed for 35 patients during hospitalization. Among 18 patients examined for epidermal growth factor receptor (EGFR) and Anaplastic lymphoma kinase (ALK) mutations, 15 were positive for mutations of these driver genes; 10 had sensitive EGFR mutations, while 5 patients had clear ALK rearrangement. Only three patients were driver gene mutation-negative (Table $3)$.

Table 3. Genetic status assay $(n=53)$

\begin{tabular}{lll}
\hline & $\mathrm{n}$ & $\%$ \\
\hline Driver gene mutation-positive & & \\
EGFR TKI-sensitive mutation & 10 & 18.87 \\
ALK rearrangement & 5 & 7.55 \\
Driver gene mutation-negative & 3 & 5.66 \\
No genetic testing during hospitalization & 35 & 66.04 \\
\hline
\end{tabular}

TKI, tyrosine kinase inhibitor

\section{Treatment}

Surgery was conducted for $47.89 \%$ of patients. Of 59 patients with radiotherapy records, 15 had undergone radiation treatment, among which nine 
underwent thoracic radiotherapy, four cranial irradiation, and two were treated with radiation targeting both thoracic and cranial sites. Chemotherapy was administered to $68.33 \%$ of patients and 14 patients received targeted drug therapy, 9 and 5 of which were administered EGFR and ALK TKIs, respectively (Table 4). More than one treatment modality (surgery, radiotherapy, chemotherapy, and targeted therapy) was applied in $41.79 \%(28 / 67)$ of patients.

Table 4. Patient treatment strategies

\begin{tabular}{lll}
\hline & $\mathrm{n}$ & $\%$ \\
\hline Surgery $(\mathrm{n}=71)$ & 34 & 47.89 \\
Yes & 37 & 52.11 \\
No & & \\
Radiotherapy $(\mathrm{n}=59)$ & 15 & 25.42 \\
Yes & 44 & 74.58 \\
No & & \\
Chemotherapy $(\mathrm{n}=60)$ & 41 & 68.33 \\
Yes & 19 & 31.67 \\
No & & \\
Targeted therapy $(\mathrm{n}=52)$ & 14 & 26.92 \\
Yes & 38 & 73.08 \\
No & &
\end{tabular}

\section{Survival Analysis}

The 1-year survival rate was $62.31 \%$, while and the 3- and 5-year survival rates were both $53.31 \%$. Median OS was not achieved (range, 3-86 months) (Figure 3).

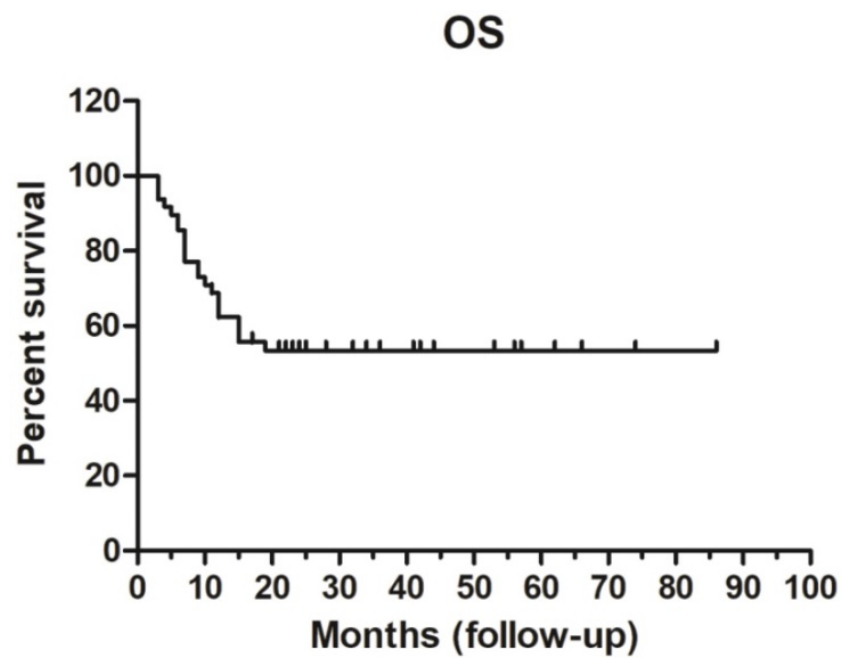

Figure 3. Overall survival (OS) curve $(n=48)$.

Sex was strongly associated with OS. Female OS (median, undefined) was superior to that of males (median OS, 13.5 months; $\mathrm{p}<0.05$ ). Patients clearly positive for driver gene mutations, such as TKI-sensitive EGFR mutations or ALK rearrangements had superior OS compare with those with negative or unknown driver gene mutation status; the latter had a median OS of only 15 months.
Stage was another important prognostic factor for OS. Unsurprisingly, patients with stage IV disease had much shorter OS than those with stage 0 -III $(p<0.01)$; median OS was only 10 months for patients with stage IV disease (Table 5; Figure 4).

Table 5. Univariate analysis of prognostic factors for OS

\begin{tabular}{|c|c|c|c|}
\hline Prognostic factor & Hazard ratio $(95 \% \mathrm{CI})$ & p-value ${ }^{a}$ & $\mathrm{p}$-value ${ }^{\mathrm{b}}$ \\
\hline $\operatorname{Sex}(\mathrm{M}: \mathrm{F})$ & $2.65(1.12-6.23)$ & 0.0258 & 0.0317 \\
\hline $\begin{array}{l}\text { Driver gene } \\
\text { (+ vs. -/unknown) }\end{array}$ & $0.41(0.16-1.01)$ & 0.0531 & 0.0351 \\
\hline Stage 0-III/IV & $4.63(1.76-12.19)$ & 0.0019 & 0.0024 \\
\hline $\begin{array}{l}\text { Pathology } \\
\text { (Adenocarcinoma vs. } \\
\text { Squamous carcinoma) }\end{array}$ & $7.44(0.98-56.46)$ & 0.0522 & 0.0394 \\
\hline $\begin{array}{l}\text { (Squamous carcinoma vs. } \\
\text { Small cell carcinoma) }\end{array}$ & $0.70(0.20-2.47)$ & 0.5833 & 0.9567 \\
\hline $\begin{array}{l}\text { (Adenocarcinoma vs. Small cell } \\
\text { carcinoma) }\end{array}$ & $0.09(0.027-0.28)$ & $<0.0001$ & 0.0001 \\
\hline
\end{tabular}

Analysis according to pathological type demonstrated that lung adenocarcinoma patients attained much longer OS than those with squamous carcinoma and small cell lung cancer (particularly those with small cell lung cancer; $p<0.01$ ). There was no difference in OS between young adults with squamous carcinoma and small cell lung cancer. The median survival rates for patients with adenocarcinoma, squamous carcinoma, and small cell lung cancer were undefined, 9 months, and 10.5 months, respectively (Table 5; Figure 4).

\section{Discussion}

With rapid increases in the morbidity and mortality attributable to lung cancer globally, research on lung cancer in young patients is attracting considerable attention; however, previous data is scant and discordant. The definition of young with regard to lung cancer is uncertain, and generally ranges from $40-50$ years [1, 5-12]. To the best of our knowledge, our study is the first to focus on much younger patients with lung cancer (aged 18-35 years), with the aim of discovering traits specifically associated with lung cancer in the young.

The incidence of early-onset lung cancer differs according to geographical region and over time. In our cohort, the incidence of lung cancer in the young population (aged 18-35 years) was $1.37 \%$, which is similar to the rate of $1.84 \%$ reported previously in patients aged 20-45 years from Beijing, China [1]. Rich et al. reported that young adults (18-39 years) accounted for $0.5 \%$ of non-small cell lung cancer (NSCLC) in the UK from 2004-2011 [6], and Subramanian et al. detected an incidence rate of $1.16 \%$ in a young population (18-40 years) in the USA, using data from the Surveillance, Epidemiology, and End 
Results (SEER) database [7]. Zhang et al. reported that early-onset NSCLC cancer accounted for $5.27 \%$ of lung cancer among patients aged $15-45$ years, based on data from 175 qualified hospitals in the greater Shanghai area [8].

We found that only $1.32 \%$ patients had a family history of lung cancer, while $28.40 \%$ were former or current smokers, which is much lower than the $26.7 \%$ and $72.4 \%$, respectively, previously reported by $\mathrm{Li}$ et al. [1], who found that smoking and family history of lung cancer were risk factors for early-onset lung cancer; however, analysis of the demographic data collected in our study failed to identify clear correlations with these factors.

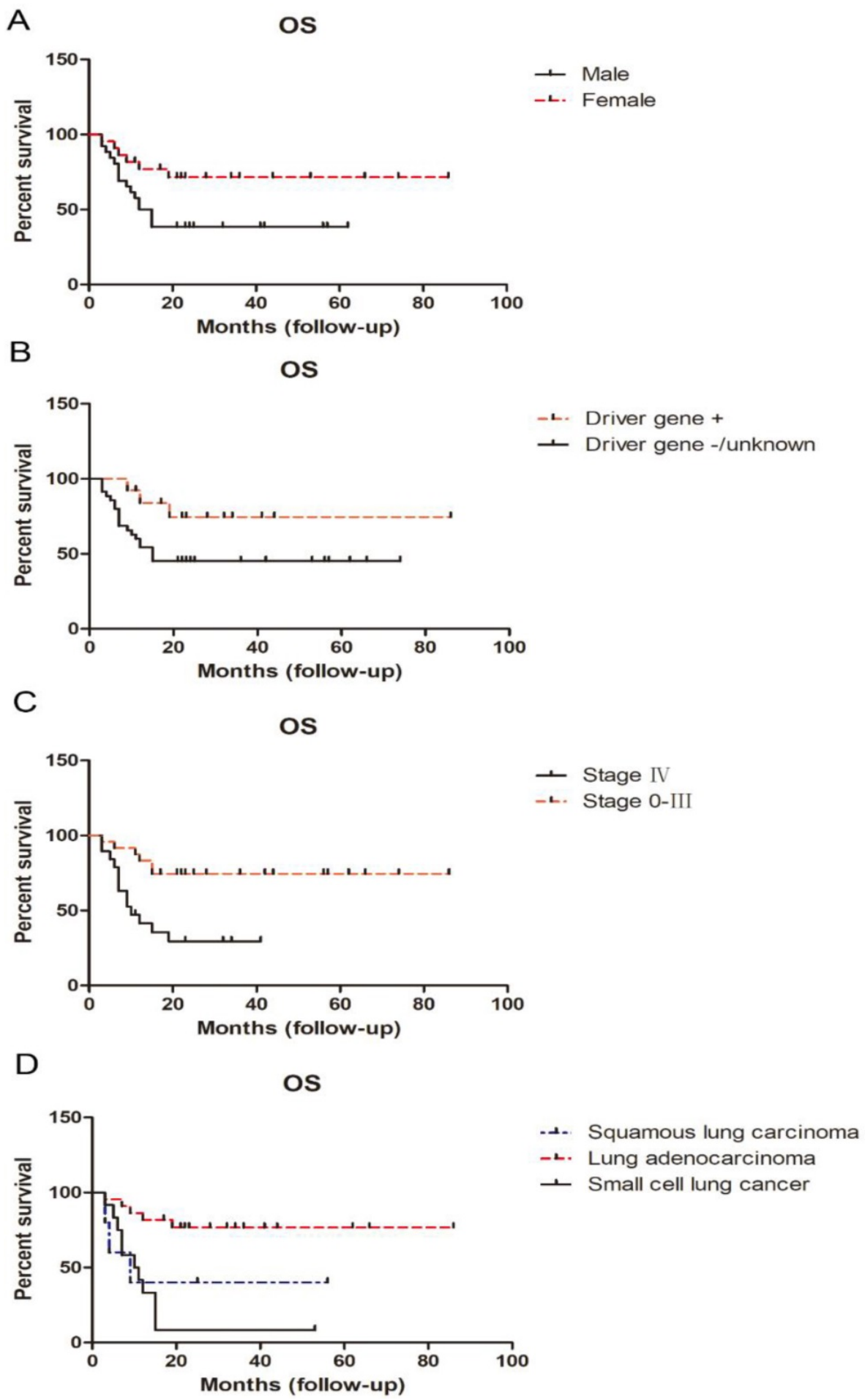

Figure 4. Influence of sex, driver gene status, stage, and pathological type on OS in young adults with lung cancer. 
Our retrospective study demonstrated that male sex, lack of detection of driver gene mutation, stage IV disease, and squamous carcinoma or small cell lung cancer pathological type, predicted dismal outcomes, in terms of OS; however, no significant difference in survival rate was found by $\mathrm{Li}$ et al. according to patient sex or pathological tumor type [1]. These results appear to be conflicting and suggest that there is considerable heterogeneity among young adults with lung cancer. Large scale, multicentric, or even national, data is urgently required to fully explore the features, best interventions, and prognosis of early-onset lung cancer.

Remarkably, almost half (49.21\%) of the cohort for whom tumor stage was recorded had metastatic disease, which is similar to previous reports; for example, metastasis was detected in $58 \%$ and $36 \%$ of patients with early onset lung cancer by Subramanian et al. [7] and Rich et al. [6], respectively. On further investigation, $68 \%$ of patients with metastases had single organ involvement, namely oligometastasis; the term "oligometastasis" was first introduced by Hellman and Weichselbaum in 1995 [13], and is generally considered a status between local and disseminated disease. Even when the same intervention strategy is applied, the number of metastatic foci determines patient prognosis to a considerable extent [14-16]. Despite the late-stage at presentation, the young patients in our study had a 5 -year survival rate of $53.31 \%$, which was higher than that documented by Duan et al. (31\%) in 2013 [17]. This phenomenon can be partly explained by the fact that a clear majority of patients had oligometastases, as described above. Additionally, young patients generally have good performance status and few physical morbidities, making it feasible for them to endure multiple aggressive treatment modalities. In our cohort, $41.79 \%$ patients underwent $\geq 2$ modalities of management, including surgery, radiotherapy, chemotherapy, and targeted therapy. Multiple treatments, with the aim of maximizing cancer control with tolerable side effects, will ultimately translate into survival benefits.

The cause of lung cancer in the young is a current research hotspot. Pan et al. conducted an analysis of patients with NSCLC aged $\leq 40$ years and found that frequent genomic alterations occur in this subset; $40 \%(29 / 73)$ of patients with adenocarcinoma harbored EGFR mutations and 34\% (25/74) had ALK rearrangements [18]. Another investigation revealed that, in patients aged $\leq 50$ years, a high proportion (59\%) had a targetable genotype [19]. Hou et al. found that, in patients with lung adenocarcinoma aged $\leq 45$ years, there was a higher frequency of human epidermal growth factor receptor 2 (HER2) and ALK genetic alterations, with concurrent EGFR/TP53 mutations, but a lower prevalence of mutations of EGFR exon 20, KRAS, and serine/threonine kinase 11 (STK11), relative to patients aged $>45$ years old [20]. In our study, only 18 patients had been screened for EGFR and ALK mutation status and 55.56\% (10/18) harbored TKI-sensitive EGFR mutations, while $27.78 \%$ (5/18) had ALK rearrangements. Despite the small sample size for genetic testing, these data suggest a high mutation rate in young patients with lung cancer. These distinct genomics profile in early-onset lung cancer have fundamental implications for practice. We propose that next-generation sequencing should be conducted for these specific patients to guide personalized management. For driver gene mutation-positive lung cancer patients, specific targeted drugs are critical for disease control and outcome [21-25].

Compared with lung cancer in other age groups, young adults (aged 18-39 years) with lung cancer are predominantly diagnosed with adenocarcinoma $(48 \%)$, advanced stage at diagnosis $(71 \% \mathrm{IIIb} / \mathrm{IV})$, good performance status $(0-1,85 \%)$, were more likely to undergo surgery and chemotherapy, and achieved better OS and postoperative OS, according to a report by Rich et al. [6]. Subramanian et al. also demonstrated that younger patients with NSCLC ( $\leq$ 40 years) were more likely to be female and have adenocarcinoma compared with older patients $(>40$ years) and, despite a higher proportion of patients with stage IV disease, the young cohort had superior OS and cancer-specific survival [7]. In a study conducted by Suidan et al., compared with older patients ( $\geq 60$ years), those with early-onset lung cancer ( $\leq 50$ years) had similar sex distribution, smoker ratio, a larger proportion of small cell lung cancer $(12 \%$ vs. $3 \%, p<0.001)$, a higher EGFR mutation rate $(18 \%$ vs. $13 \%, \mathrm{p}=0.06)$, a higher ALK translocation rate $(9 \%$ vs. $1 \%, \mathrm{p}<0.001)$, more brain metastases $(38 \%$ vs. $21 \%, \mathrm{p}=0.002)$, and more frequently underwent targeted therapy (25\% vs. $16 \%$, $\mathrm{p}=0.015)$. Following further stratification, patients < 40 years old appeared to exhibit superior OS (70.8 months, $p=0.05$ ). Further, the presence of driver mutations improved the survival prognosis for young individuals (31.9 vs. 17.0 months, $p=0.003$ ) [26].

Our study has several limitations. First, it was a single-center retrospective investigation. Further, a relatively small proportion of our patients underwent genetic assessment. In the future, larger-scale and deeper investigation of this topic will be initiated.

\section{Conclusions}

Lung cancer in the young population is a distinct entity that predominantly presents as 
adenocarcinoma, with late-stage at onset, and a higher frequency of gene alteration, relative to older patients. Male sex, lack of driver gene mutation, stage IV disease, and squamous carcinoma or small cell lung cancer pathological types are associated with dismal outcomes in young adults. Genetic assessment is imperative for clinical management of this subgroup, to determine driver gene mutation status and guide application of targeted therapies. Despite the frequency of late-stage disease at diagnosis in young patients, the higher proportion of oligometastasis in this group highlights the importance of intensive treatment for improving patient prognosis. Our study is the first to focus on much younger patients with lung cancer (aged 18-35 years). To overcome the limitations of retrospective research and the considerable heterogeneity of patients with early-onset lung cancer, multi-center, or even national, databases should be established to identify the properties and optimal treatment paradigms for this unique entity in the near future.

\section{Acknowledgements}

This study was supported by a research program from the National Natural Science Foundation of China (grant no. 81602659).

\section{Competing Interests}

The authors have declared that no competing interest exists.

\section{References}

1. Li J, Yang F, Li X, et al. Characteristics, survival, and risk factors of Chinese young lung cancer patients: the experience from two institutions. Oncotarget. 2017; 8:89236-89244.

2. Lan L, Zhao F, Cai Y, et al. [Epidemiological analysis on mortality of cancer in China, 2015]. ZhonghuaLiu Xing Bing Xue Za Zhi. 2018; 39:32-34.

3. Strand TE, Malayeri C, Eskonsipo PK, et al. Adolescent smoking and trends in lung cancer incidence among young adults in Norway 1954-1998. Cancer Causes Control. 2004; 15:27-33.

4. Marugame T, Yoshimi I, Kamo K, et al. Trends in lung cancer mortality among young adults in Japan. Jpn J Clin Oncol. 2005; 35:177-80.

5. Liu M, Cai X, Yu W, et al. Clinical significance of age at diagnosis among young non-small cell lung cancer patients under 40 years old: a population-based study. Oncotarget. 2015; 6:44963-70.

6. Rich AL, Khakwani A, Free CM, et al. Non-small cell lung cancer in young adults: presentation and survival in the English National Lung Cancer Audit. QJM. 2015; 108:891-7.

7. Subramanian J, Morgensztern D, Goodgame B, et al. Distinctive characteristics of non-small cell lung cancer (NSCLC) in the young: a surveillance, epidemiology, and end results (SEER) analysis. J Thorac Oncol. 2010; 5:23-8.

8. Zhang J, Chen SF, Zhen Y, et al. Multicenter analysis of lung cancer patients younger than 45 years in Shanghai. Cancer. 2010; 116:3656-62.

9. Hanagiri T, Sugio K, Uramoto H, et al. Results of surgical treatment for lung cancer in young adults. Int Surg. 2008; 93:50-4.

10. Mauri D, Pentheroudakis G, Bafaloukos D, et al. Non-small cell lung cancer in the young: a retrospective analysis of diagnosis, management and outcome data. Anticancer Res. 2006; 26:3175-81.

11. Yang P, Bamlet WR, Ebbert JO, et al. Glutathione pathway genes and lung cancer risk in young and old populations. Carcinogenesis. 2004; 25:1935-44.

12. Gomes $\mathrm{R}$, Dabo $\mathrm{H}$, Queiroga $\mathrm{H}$, et al. Non-small cell lung cancer in young patients--a retrospective analysis of 10 years in a tertiary university hospital. Rev Port Pneumol (2006). 2016; 22:125-6.

13. Hellman S, Weichselbaum RR. Oligometastases. J Clin Oncol. 1995; 13:8-10.

14. Suzuki H, Yoshino I. Approach for oligometastasis in non-small cell lung cancer. Gen Thorac Cardiovasc Surg. 2016; 64:192-6.
15. Krebs MG, Sloane R, Priest L, et al. Evaluation and prognostic significance of circulating tumor cells in patients with non-small-cell lung cancer. J Clin Oncol. 2011; 29:1556-63.

16. Salama JK, Chmura SJ, Mehta N, et al. An initial report of a radiation dose-escalation trial in patients with one to five sites of metastatic disease. Clin Cancer Res. 2008; 14:5255-9.

17. Duan $\mathrm{L}$, You $\mathrm{Q}$, Chen $\mathrm{X}$, et al. Outcome and prognosis for patients younger than thirty with primary lung cancer. Minerva Chir. 2013; 68:175-82.

18. Pan X, Lv T, Zhang F, et al. Frequent genomic alterations and better prognosis among young patients with non-small-cell lung cancer aged 40 years or younger. Clin Transl Oncol. 2018; 20:1168-1174.

19. Sacher AG, Dahlberg SE, Heng J, et al. Association Between Younger Age and Targetable Genomic Alterations and Prognosis in Non-Small-Cell Lung Cancer. JAMA Oncol. 2016; 2:313-20.

20. Hou H, Zhu H, Zhao H, et al. Comprehensive Molecular Characterization of Young Chinese Patients with Lung Adenocarcinoma Identified a Distinctive Genetic Profile. Oncologist. 2018; 23:1008-1015.

21. Yang JC, $\mathrm{Wu} \mathrm{YL}$, Schuler $\mathrm{M}$, et al. Afatinib versus cisplatin-based chemotherapy for EGFR mutation-positive lung adenocarcinoma (LUX-Lung 3 and LUX-Lung 6): analysis of overall survival data from two randomised, phase 3 trials. Lancet Oncol. 2015; 16:141-51.

22. Rosell R, Carcereny E, Gervais R, et al. Erlotinib versus standard chemotherapy as first-line treatment for European patients with advanced EGFR mutation-positive non-small-cell lung cancer (EURTAC): a multicentre, open-label, randomised phase 3 trial. Lancet Oncol. 2012; 13:239-46.

23. Douillard JY, Ostoros G, Cobo M, et al. First-line gefitinib in Caucasian EGFR mutation-positive NSCLC patients: a phase-IV, open-label, single-arm study. Br J Cancer. 2014; 110:55-62.

24. Peters S, Camidge DR, Shaw AT, et al. Alectinib versus Crizotinib in Untreated ALK-Positive Non-Small-Cell Lung Cancer. N Engl J Med. 2017; 377:829-838.

25. Solomon BJ, Mok T, Kim DW, et al. First-line crizotinib versus chemotherapy in ALK-positive lung cancer. N Engl J Med. 2014; 371:2167-77.

26. Suidan A, Ilouze M, Peled N. Lung Cancer in Young Patients: Higher Rate of Driver Mutations, Brain Involvement and Better Survival. J Thorac Oncol. 2017; 12(Suppl 1):S1526. 